\title{
The hazards of excessive folic acid intake in MTHFR gene mutation carriers: An obstetric and gynecological perspective
}

\author{
Laura Tafuri $^{1 *}$, Edouard J Servy ${ }^{1}$ and Yves J R Menezo ${ }^{2}$ \\ ${ }^{1}$ The Servy Massey Institute, 812 Chafee Ave, Augusta, GA, USA \\ ${ }^{2}$ Laboratoire Clement, Paris, France and London Fertility associates, Harley St, London UK
}

For decades, Folate has been synonymous with reproductive health. Not only is it important for oogenesis and spermatogenesis, but in the prevention of neural tube defects. Folic acid can be found in many foods naturally like kale, spinach, arugula and swiss chard, but is also in synthetic form in certain flours, starches, and prenatal vitamins that have been supplemented with it. But how much is too much?

It was assumed that when someone wishes to get pregnant, and is successful, that folic acid must be taken everyday religiously. However, with recent developments in the world of infertility, this assumed "holy grail" supplement is actually being shown to have more risks than benefits. This is in particularly large doses, around $4-5 \mathrm{mg}$, and in certain patients with the Methylene Tetrahydrofolate Reductase (MTHFR) Single Nucleotide Polymorphisms (SNPs)

MTHFR is the enzyme involved not only in the folate cycle, but also crucial to the 1-carbon cycle (1-CC), responsible for methylation. Methylation is essential for the regeneration and biochemical regulation of cells, imprinting genes and epigenetics. In addition, it aids in processing amino acids for neurotransmitters, detoxification of compounds like Homocysteine (Hcy), and in DNA repair. However, when the MTHFR SNP is present, this external supply of synthetic folic acid that is unable to be fully processed, accumulates and can actually become hazardous. The cofactor S-Adenosyl Methionine (SAM) is fundamental for methylation and once the targeted cell gets methylated, S-Adenosyl homocysteine (SAH) is formed (Figure 1). Hydrolysis then occurs and releases Hcy that must be recycled via the 1 carbon cycle to prevent this toxic compound for accumulating. Hcy works as an inhibitor of the methylation processes via a negative feedback mechanism. This step is critical, as a build up of homocysteine not only has the ability to damage blood vessels via accelerated atherosclerosis, but creates a hypercoaguable state that can form clots leading to early fetal loss and repeated first trimester miscarriages.

The cycle above demonstrates how the MTHFR enzyme takes the natural folic acid derivates from the diet or synthetic folic acid supplements we ingest and transforms it to 5-Methyl-THF, the active form that is ready for use by our cells. By taking 5-methyl-THF supplements, the mutated MTHFR is bypassed and cells can still get the active enzymes needed for those crucial processes. In addition, by not taking synthetic folic acid supplements and ingesting more of the naturally active folate through leafy greens, it will not build up and cause the detrimental clots in the blood responsible for miscarriages.

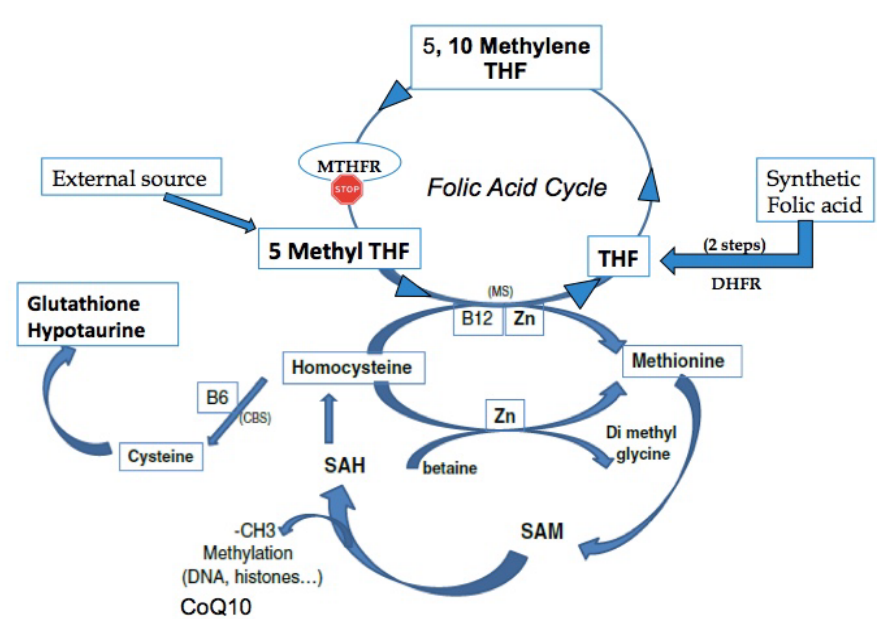

Figure 1. MTHFR SNPs and folic acid metabolism; impact on the one carbon cycle and methylation processes. Without a correct and efficient activity of MTHFR. Folic acid consumption is useless and hazardous for health: It cannot feed the 1-CC.

This active form 5-methyl-THF can participate in the gene activation and DNA repair permanently needed, as well as a growing embryo during rapid cell growth regulation and division.

One-third of the world's population is estimated to be carriers of these SNPs, with 10\% thought to be affected. The Hispanic and Caucasian population have the highest prevalence with $48 \%$ and $45 \%$ respectively carriers of the $\mathrm{C677T}$ heterozygous mutation, while African Americans have a lower prevalence of $24 \% .{ }^{1}$ There is also a higher population in southern Italy, where the frequency of homozygous mutations $\mathrm{T} 677 \mathrm{~T}$ and $\mathrm{C} 1298 \mathrm{C}$ are $25 \%$ and $12.5 \%$ respectively [2]. The amount of enzymatic activity that is decreased depends on the isoform. Of the homozygous mutations most studied, a carrier of the homozygous $\mathrm{T} 677 \mathrm{~T}$ isoform produces $75 \%$ less methyl folate than someone without the mutation, and carriers of the homozygous C1298C isoform produces 17\% less methyl folate (Table 1).

Correspondence to: Laura Tafuri, The Servy Massey Institute, 812 Chafee Ave, Augusta, GA, 30904, USA

Received: March 14, 2018; Accepted: March 23, 2018; Published: March 27, 2018 
Table 1. MTHFR gene polymorphisms [1].

\begin{tabular}{|c|c|c|c|}
\hline Normal & Heterozygous & Homozygous & $\begin{array}{c}\text { Compound } \\
\text { Heterozygous }\end{array}$ \\
\hline C677C & C677T & T677T & A1298C/C677T \\
\hline A1298A & A1298C & C1298C & C677T/A1298C \\
\hline
\end{tabular}

Many Physicians prescribe 4-5mg of folate QD in patients who are trying to, or have conceived. In addition to the prenatal vitamin supplements, synthetic folic acid is combined with many flours and grains we purchase. The problem here in lies, this excessive folate accumulates and is now recognized to be harmful. An increase in unmetabolized folic acid can lead to many issues besides fetal loss. First, it can lead to an immune dysfunction by dysregulation of natural killer cells $[3,4]$. This excess folate decreases their innate cytotoxic effects leading to increased tumorgenesis. Increased folic acid has been linked to accelerations of leukemias, colorectal and prostate carcinomas $[5,6]$. In a recent large clinic trial in the US and Canada, a $1 \mathrm{mg}$ supplement of folate was given to patients with a history of colorectal cancer. At the 3-5 year follow up mark, there was found to be $67 \%$ of increased risk of invasive colorectal adenomas or advancement of previous lesions, as well as a more than $2 \mathrm{x}$ increased risk of at least 3 colorectal adenomas and prostate cancer [3].

Failures of reproduction are also attributed to these mutations. Studies have shown that some cases of premature ovarian failure may be due to abnormal methylation, and patients should be evaluated not only with an Anti-Müllerian hormone level and ultrasound, but for MTHFR mutations as well. The MTHFR SNPs induce strong chromosomal anomalies as early as the preimplantation stages of development [7]. Hypomethylation during trophoblastic development due to this defective enzyme is another reason believed to contribute to recurrent early fetal loss [2]. Male partners should also be tested for MTHFR, as this mutation can affect not only sperm morphology and DNA quality but contribute to oligo-asthenospermia. In our Georgia centre, 22 patients with recurrent miscarriages, failed IUI and/ or IVF attempts were noted and tested for MTHFR mutations. They tested positive, either homozygous or heterozygous for the MTHFR mutations, and were started on 800mcg QD of 5-methyl THF. Upon follow up, $100 \%$ of these patients conceived successfully, many within only 2-3 months of starting the supplement. Another study out of France looked at 30 couples with infertility lasting 3-5 years, where at least one member was a carrier of one main isoforms (C677T and A1298C), who were treated with 500-800mcg QD of 5-methyl THF for 2-4 months. Of this group, 12 conceived spontaneously and 15 others with the help of assisted reproductive therapy, with the remaining still under treatment [8].

In addition, a known factor in pharmacology, such an excessive amount of folate can actually compete with and block the binding of all folate to natural receptors, resulting in down regulation. This leads to a "Pseudo-MTHFR" mutation, causing the same issues (especially with an elevated blood Hcy), as a person who may be homozygous and completely lacking the MTHFR enzyme [9]. This is especially important in patients being treated with anti-folate chemotherapy drugs like methotrexate, and are supplemented with folic acid. However, in large amounts it defeats the purpose of the drug by inducing the removal of its target, giving the malignant cells time to invade and spread.

In conclusion, Folic acid should no longer be considered a "onesize-fits-all” supplement. More ingestion of leafy greens like kale, spinach, arugula and swiss chard that provide natural folate forms, including 5-methyl-THF, should be encouraged, while synthetic forms in vitamins and starch additives should be monitored. This is especially important in those carrying the MTHFR SNPs. In these patients, it is imperative to use the active 5-methyl-THF derivative, in a dosage of $600-800 \mathrm{mcg}$ QD, to bypass the mutated enzyme and receive the complete benefits of folic acid. Therefore, the replacement of folic acid with 5-methyl-THF in prenatal vitamins should be strongly considered as universally beneficial.

\section{Conflict of Interest}

The authors declare no conflict of interest.

\section{References}

1. Servy E, Menezo Y (2017) The Methylene Tetrahydrofolate Reductase (MTHFR) Isoform Challenge. High doses of folic acid are not a suitable option compared to 5 methyl tetrahydrofolate treatment. Clin Obstet Gynecol Reprod Med: 3: 4-5.

2. Zappacosta B, Romano L, Persichilli S, Cutrone LA, Graziano M, et al. (2009) Genotype Prevalence and Allele Frequencies of 5,10-Methylenetetrahydrofolate Reductase (MTHFR) C677T and A1298C Polymorphisms in Italian Newborns. Lab Med 12: 732-736,

3. Ledowsky C (2017) The folic acid vs 5-MTHF Debate. MTHFRSupport Australia. https://www.mthfrsupport.com.au/folic-acid-vs-5-mthf-debate.

4. Sawaengsri H, Wang J, Desautels N (2013) Natural killer cell cytotoxicity is reduced in aged female mice fed a high folic acid diet. The FASEB J 27: 643.

5. Farber S, Cutler EC, Hawkins JW, Harrison JH, Peirce EC $2^{\text {nd }}$, et al. (1947) The Action of Pteroylglutamic Conjugates on Man. Sci 106: 619-621. [Crossref]

6. Kim YI (2008) Folic acid supplementation and cancer risk: point. Cancer Epidemiol Biomarkers Prev 17: 2220-2225. [Crossref]

7. Enciso M, Sarasa J, Xanthopoulou L, Bristow S, Bowles M, et al. (2016) Polymorphisms in the MTHFR gene influence embryo viability and the incidence of aneuploidy. Hum Genet 135: 555-568. [Crossref]

8. Servy E, Jacquesson-Fournols L, Cohen M (2018) MTHFR isoform carriers, 5 MTHF (5Methyl TetraHydrofolate) vs Folic acid: a key to gamete quality and pregnancy outcome.

9. Xia W, Hilgenbrink AR, Matteson EL, Lockwood MB, Cheng JX, et al. (2009) A functional folate receptor is induced during macrophage activation and can be used to target drugs to activated macrophages. Blood 113: 438-446. [Crossref]

Copyright: (C2018 Tafuri L. This is an open-access article distributed under the terms of the Creative Commons Attribution License, which permits unrestricted use, distribution, and reproduction in any medium, provided the original author and source are credited. 